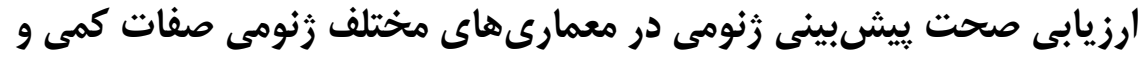

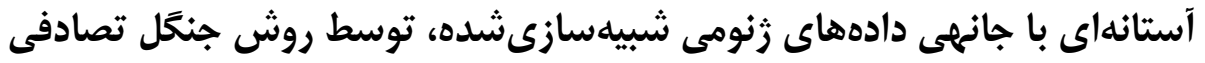

\author{
يوسف نادرى
}

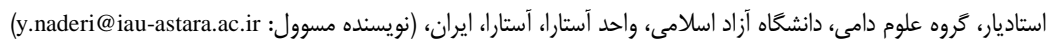

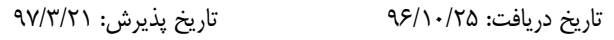

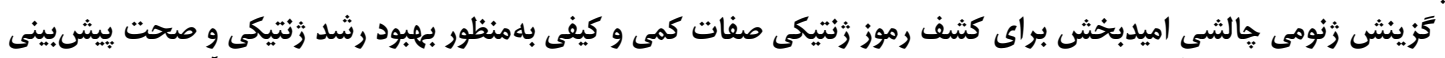

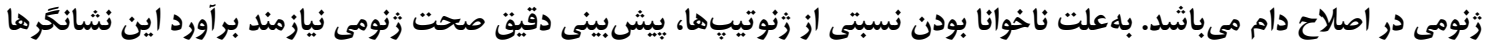

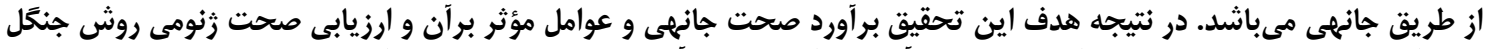

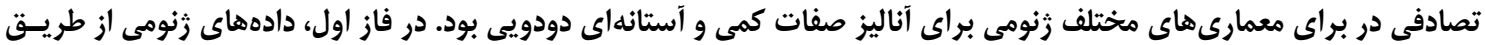

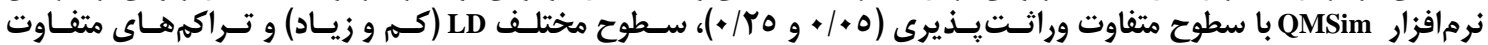

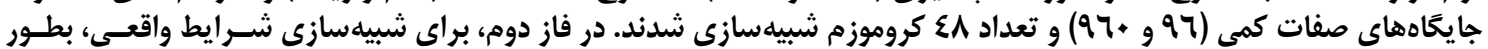

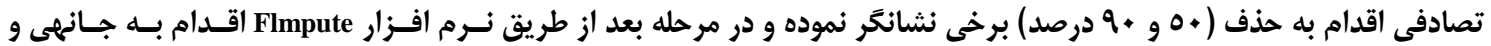

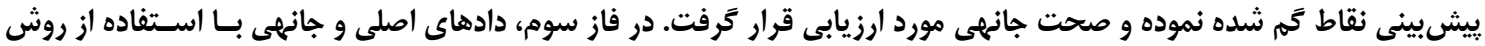

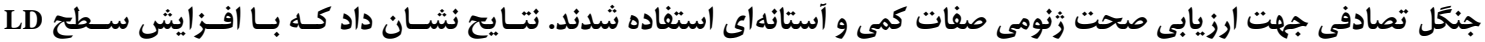

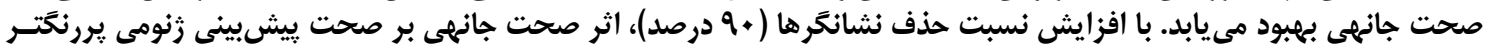

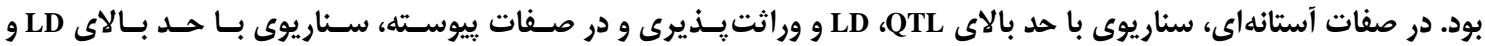

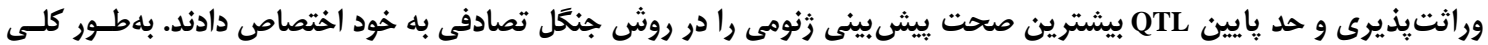

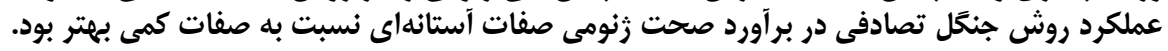

وازههاى كليدى: عدم تعادل ييوستكى، صفات آستانهاى، يادَّيرى ماشينى، جانهى، معمارى زنومى

مى كند، در حاليكه دادهاى شبيهسازى شده بله محقـق اجـازه

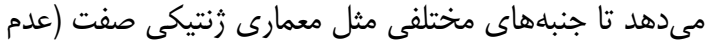

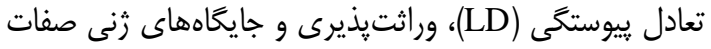

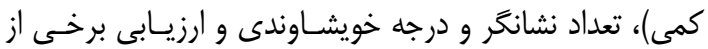

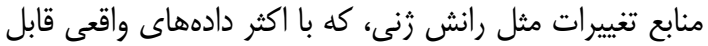

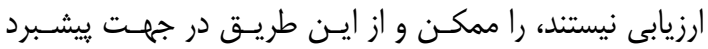

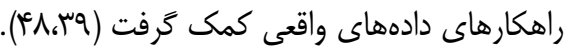

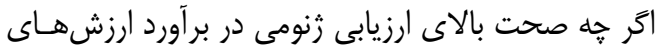

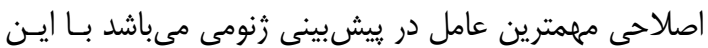

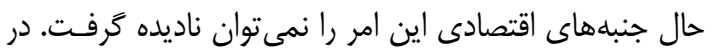

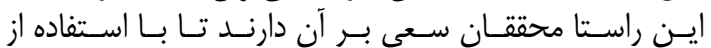

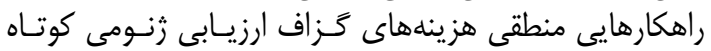

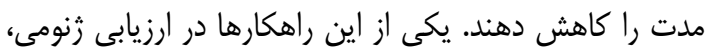

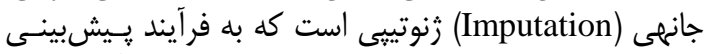

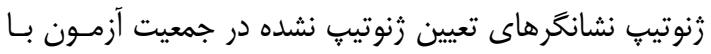

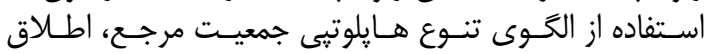

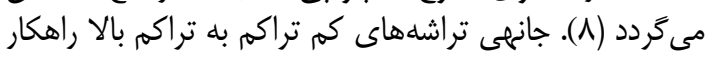

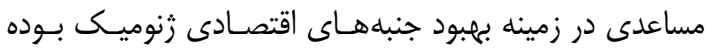

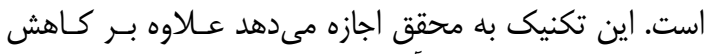

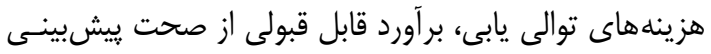

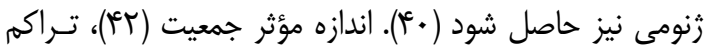

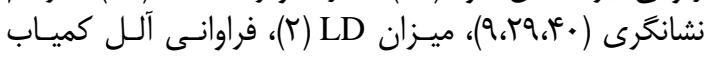

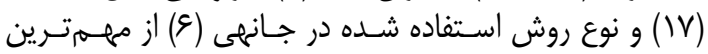

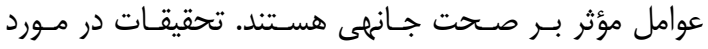

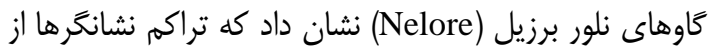

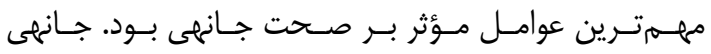

هدف اصلى در برنامههاى اصلاح دام بهبـود خصوصـيات

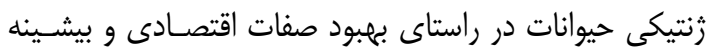

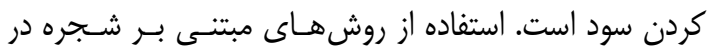

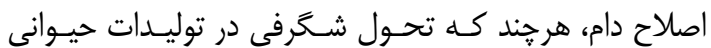

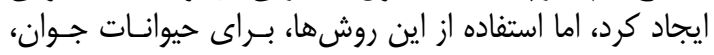

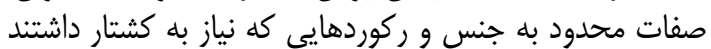

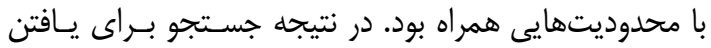

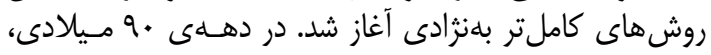

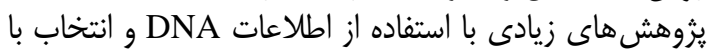

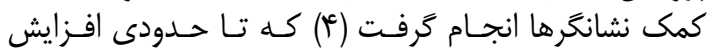

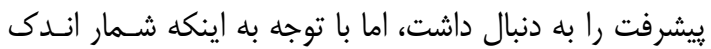

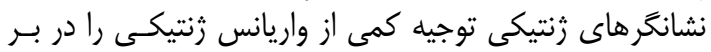

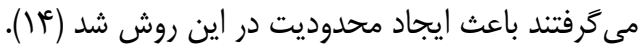

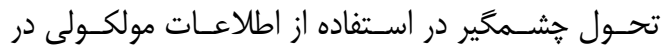

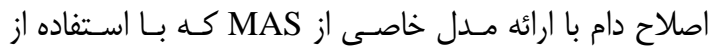

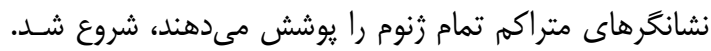

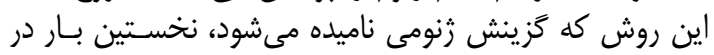

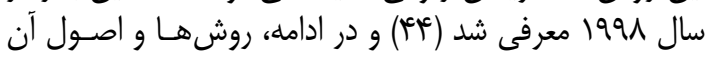

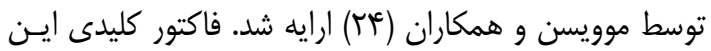

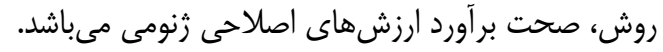

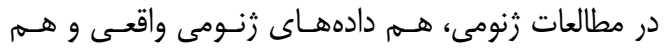

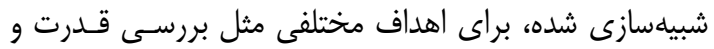

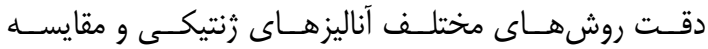

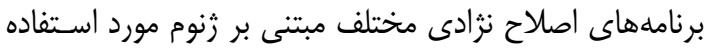

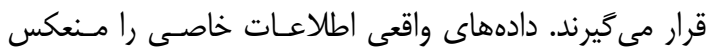


كرفته شد. نوع سيستم تلاقى تصادفى بـود و بـراى • • نسـل

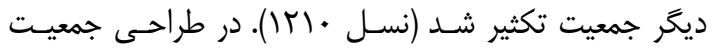

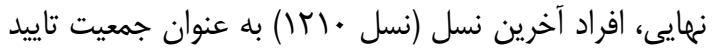

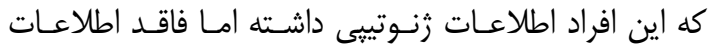

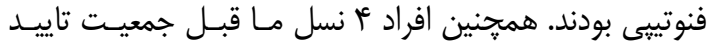

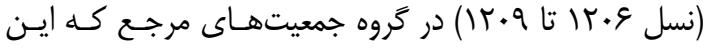

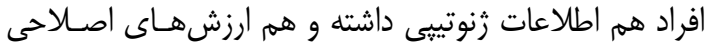

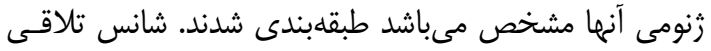

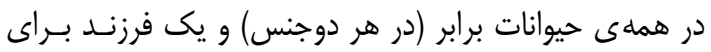

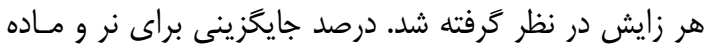

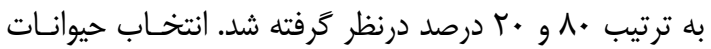

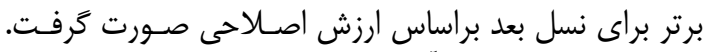

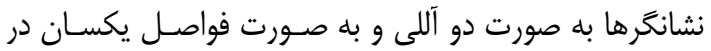

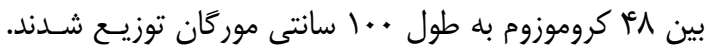

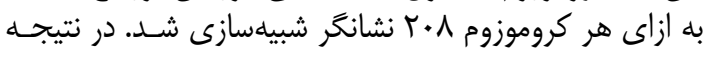

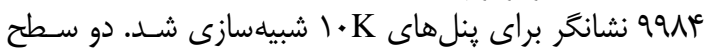

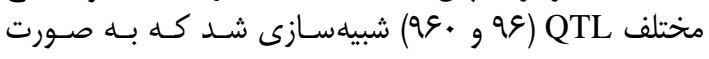

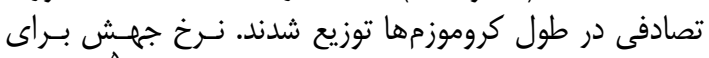

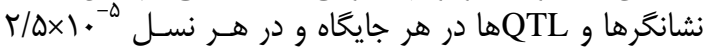

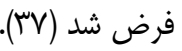

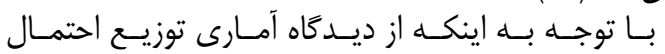

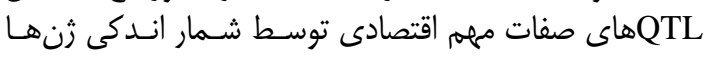

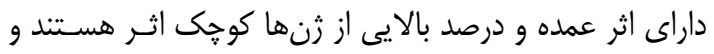

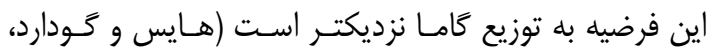

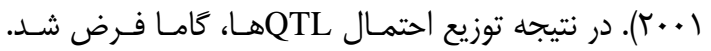

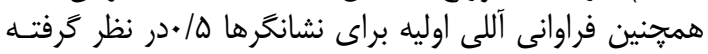

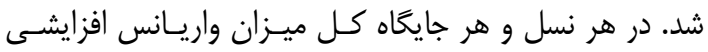

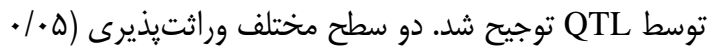

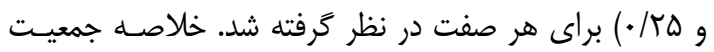

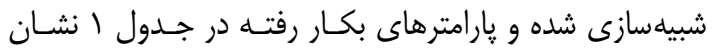

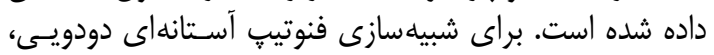

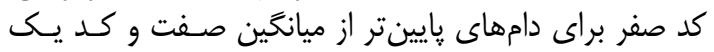

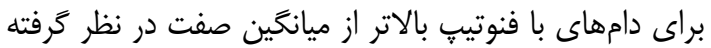

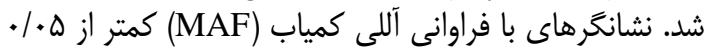

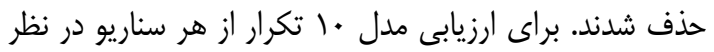

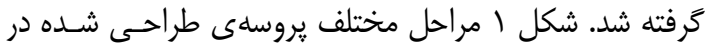

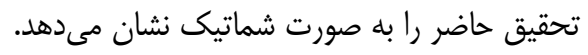

جانهمى نشانكر ها لها

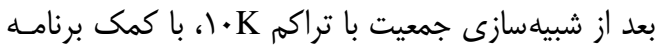

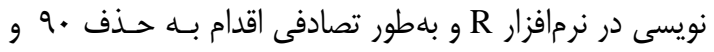

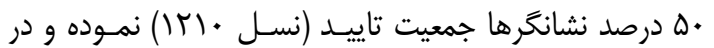

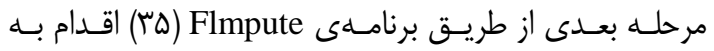

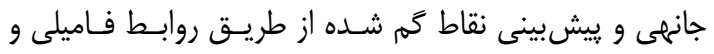

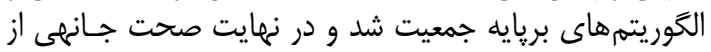

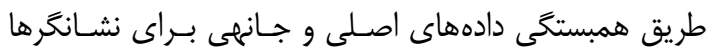

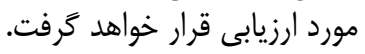
عدم تعادل بييوستكى

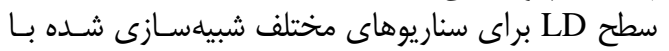

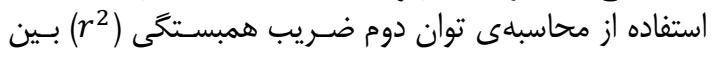

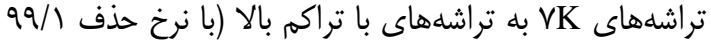

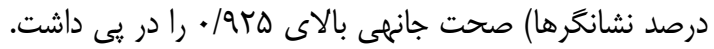

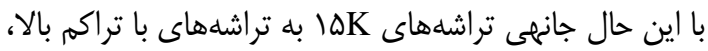

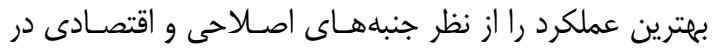

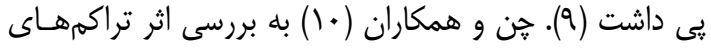

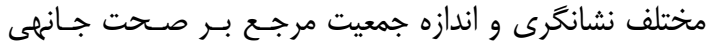

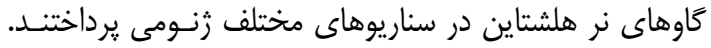

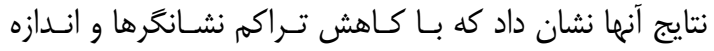

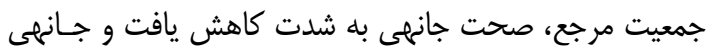

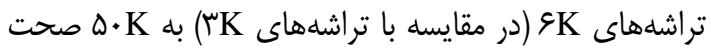

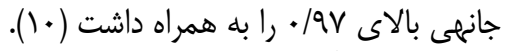

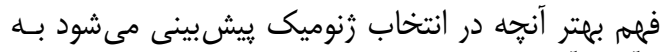

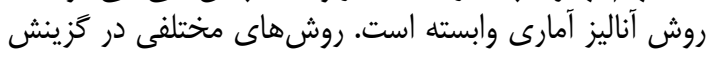

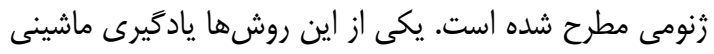

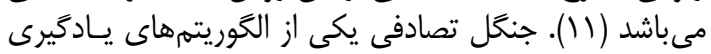

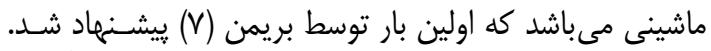

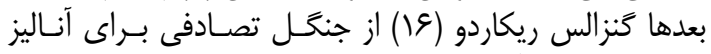

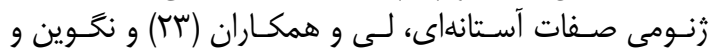

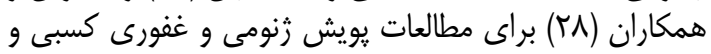

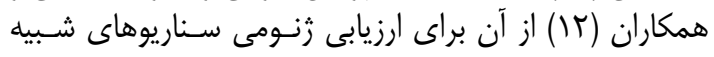

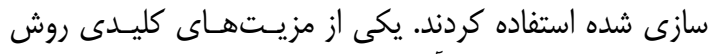

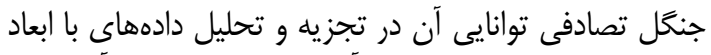

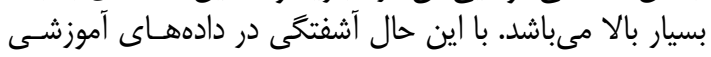

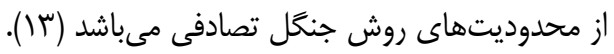

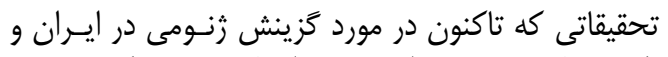

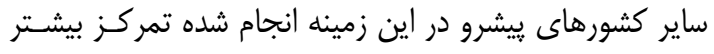

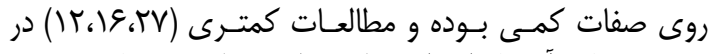

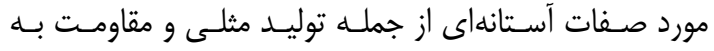

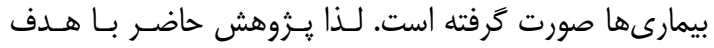

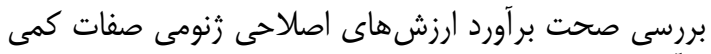

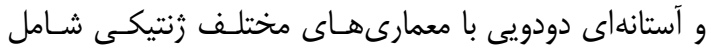

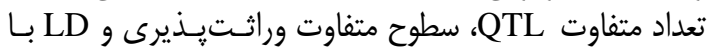

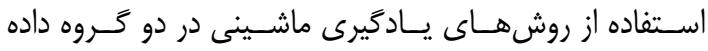
شبيهسازى شده و جانهى، انجام شده است.

\section{مواد و روشها} شبيهسازى زنوم

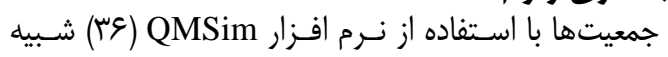

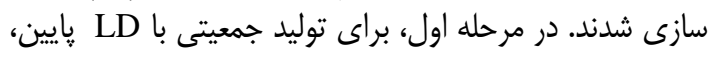

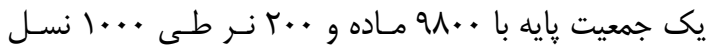

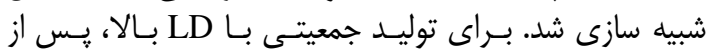

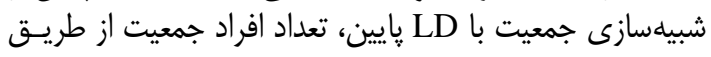

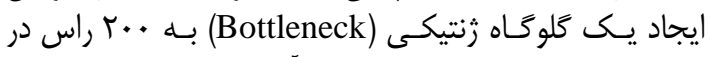

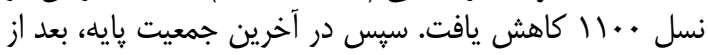

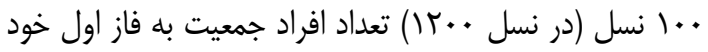

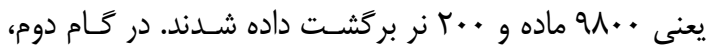

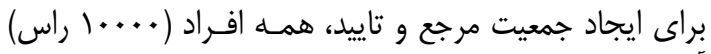

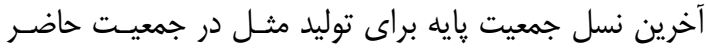

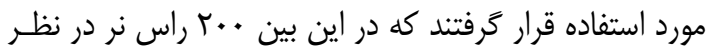




$$
\hat{f}_{r f}^{P}(x)=\frac{1}{P} \sum_{p=1}^{P} T(x ; \Psi p)
$$

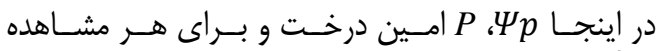
مئ مى شود.

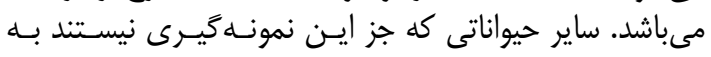

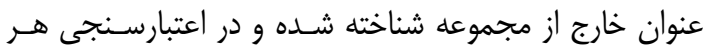

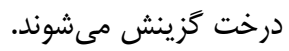

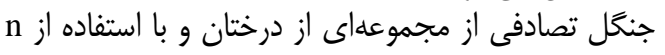

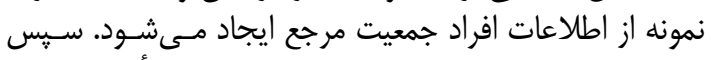

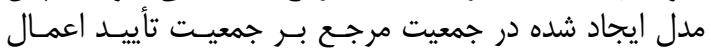

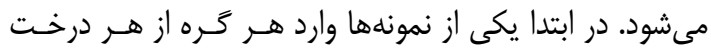

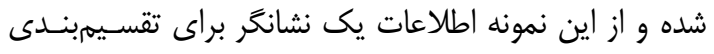

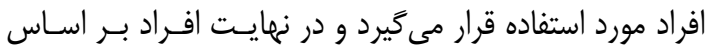

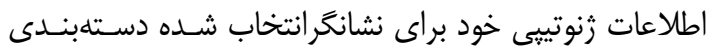

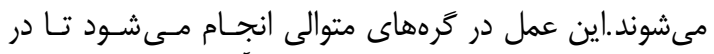

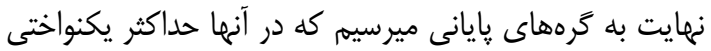

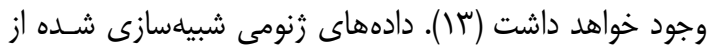
طريق بستهى RanFoG (צا) و نرمافزار R مورد آنـاليز قـرار كرفتند.

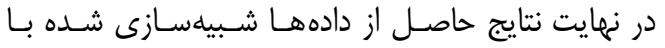

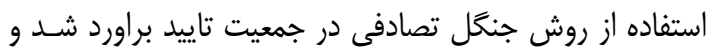

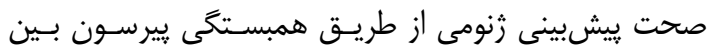

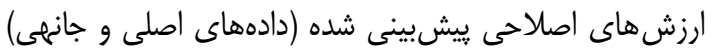

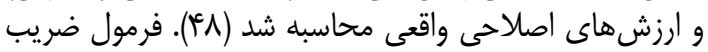

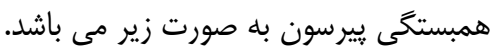
$r_{E B V ; T B V}=\frac{\sum_{i=1}^{n}(\overline{E B V}-\overline{E B V})(T B V-\overline{T B V})}{\sqrt{(\overline{E B V}-\overline{E B V})^{2}} \sqrt{(T B V-\overline{T B V})^{2}}}$

در اينجـــ

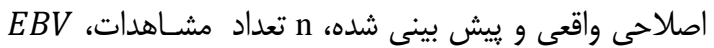

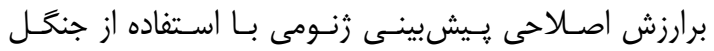

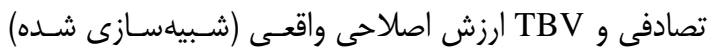

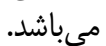

هملى جفت نشانكَرهاى ممكن ارزيابى كردد (IV): $r^{2}=\frac{D^{2}}{f(A) f(a) f(B) f(b)}$

در ايسـن فرمـــــ

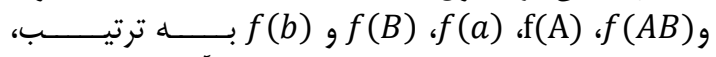

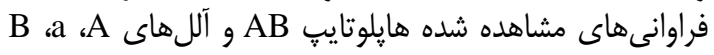

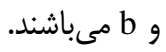

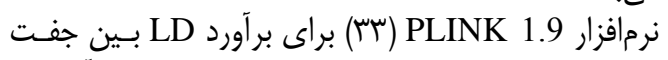

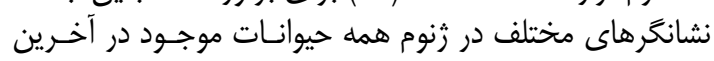
نسل مورد استفاده قرار كَرفت.

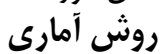

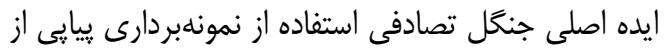

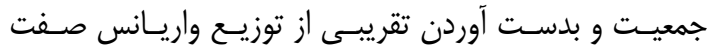

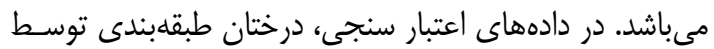

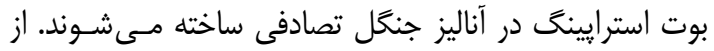

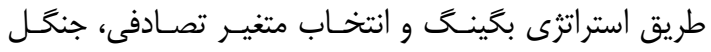

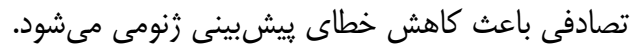

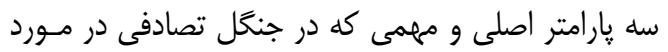

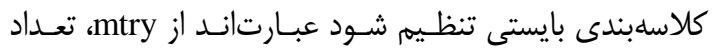

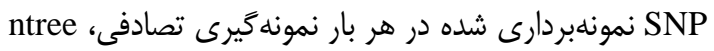

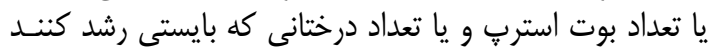

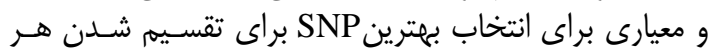

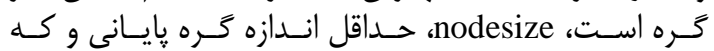
نشاندهندهى تعداد مشاهدات در هر شاخه درخت التهات

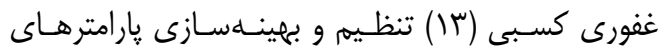

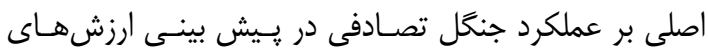

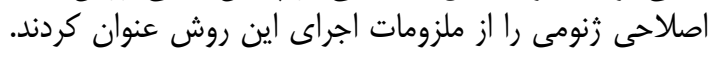

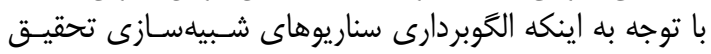

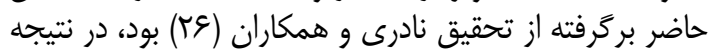

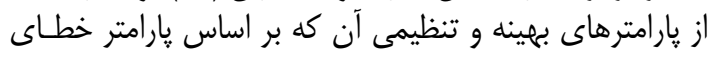

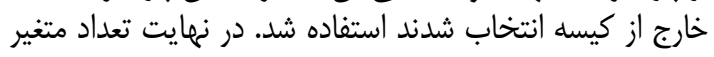

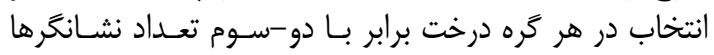

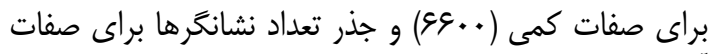

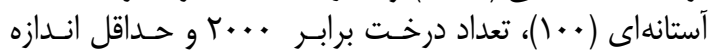

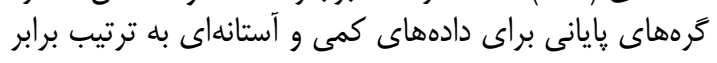

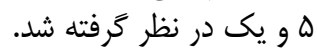
مدل كلى جنگل تصادفى به صورت زير است. 
Table 1. Parameters of the simulation process

جدول (- يارامترهاى فر آيند شبيهسازى

\begin{tabular}{|c|c|c|}
\hline LD & بالا LD* & ساختار جمعيت \\
\hline & & جمعيت اوليه \\
\hline $1 \cdots(1 \cdots \cdots)$ & $1 \cdots(1 \cdots \cdot)$ & فاز اول تعداد نسل (تعداد افراد) \\
\hline خير & بله بله & كلوكاه \\
\hline- & $11 \cdot \cdot(r \cdot \cdot)$ & فاز دوم تعداد نسل (تعداد افراد) \\
\hline- & $\mid r \cdot \cdot(1 \cdots \cdot)$ & فاز سوم تعداد نسل (تعداد افراد) \\
\hline \multicolumn{2}{|c|}{$1 \ldots$} & تعداد حيوانات در نسل آخر \\
\hline \multicolumn{2}{|c|}{ r.. } & تعداد نرهاى در نسل اخير \\
\hline \multicolumn{2}{|c|}{ १५.. } & 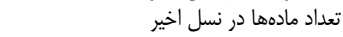 \\
\hline \multicolumn{2}{|c|}{1} & تعداد تكثيرى جمعيت اخيربعد از نسل .. Ir \\
\hline \multicolumn{2}{|c|}{ 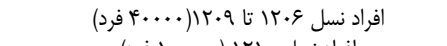 } & 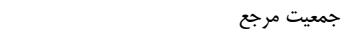 \\
\hline \multicolumn{2}{|c|}{ افراد نسل • •آ| (.... افرد) } & جمعيت تاييد \\
\hline \multicolumn{2}{|c|}{1} & تعداد نتايج به ازاى هر زايش \\
\hline \multicolumn{2}{|c|}{$\cdot / \Delta$} & احتمال نر بودن نتاج \\
\hline \multicolumn{2}{|c|}{ تصادفى } & انتخاب و طرح آميزش \\
\hline \multicolumn{2}{|c|}{$\%$. } & نرخ جايكز ينى براى نرها \\
\hline \multicolumn{2}{|c|}{$\%$} & نرخ جايكزينى براى مادهها \\
\hline \multirow{2}{*}{\multicolumn{2}{|c|}{ سن بالا/ ارزش اصلاحى برآوردى }} & معيار حذف \\
\hline & & 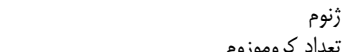 \\
\hline \multirow{2}{*}{\multicolumn{2}{|c|}{$\cdots$}} & طول هر كروموزووم (سانتي موركان) \\
\hline & & تعداد مر بو به ازاى هر كروموزوم \\
\hline \multicolumn{2}{|c|}{ كاما (c/ (•) } & QTL اثر آل هاى \\
\hline \multicolumn{2}{|c|}{ 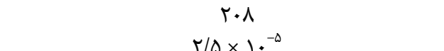 } & تعداد نشانكر به ازاى هر كروموزوم \\
\hline \multirow{2}{*}{\multicolumn{2}{|c|}{$\begin{array}{l}r / \Delta \times 1 . \Delta \\
. / r \Delta, \cdot / \cdot \Delta\end{array}$}} & نرخ جهش در نشانكر و ها \\
\hline & & وراثتيذيرى \\
\hline
\end{tabular}

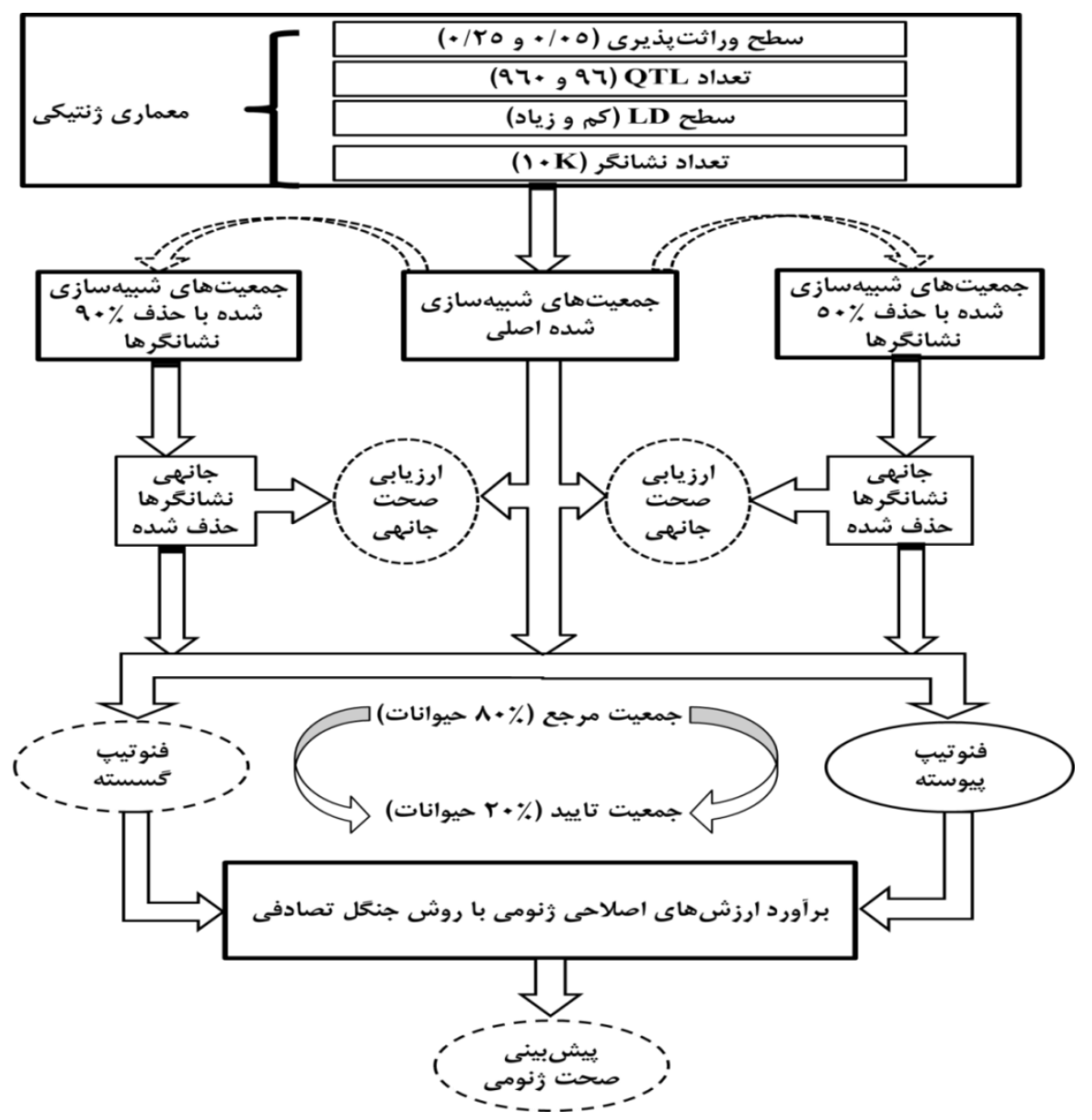

شكل ا- طرح شماتيك استراتزى كل تحقيق

Figure 1. Schematic of the whole process 


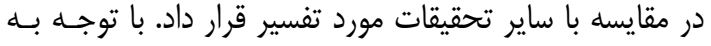

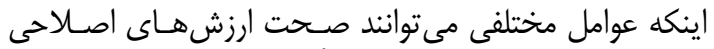

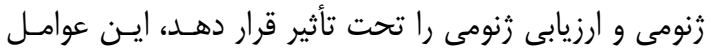

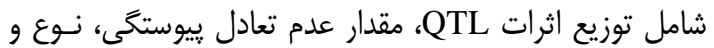

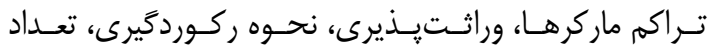

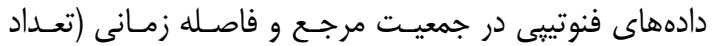

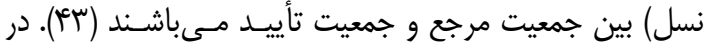

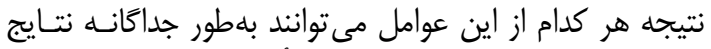
حاصل از تحقيقات مختلف را تحت تأثن تأثير قرار دهد.

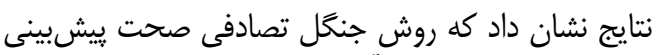

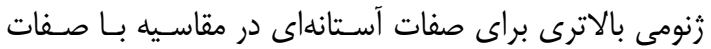

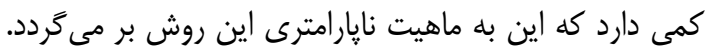

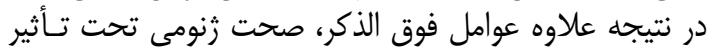

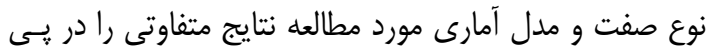

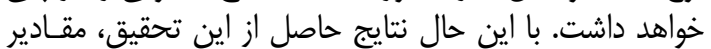

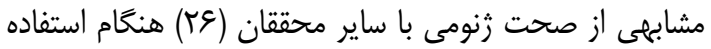

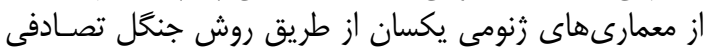

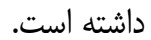

نتايج و بحث صحت جانهى و عدم تعادل بيوستَّى

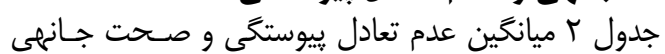

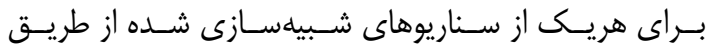

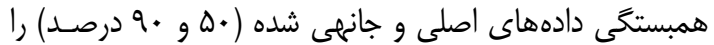

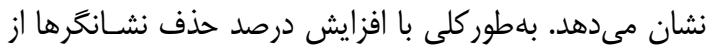

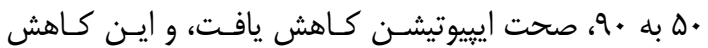

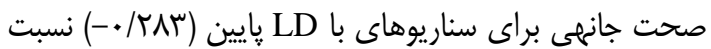

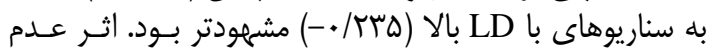

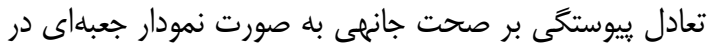

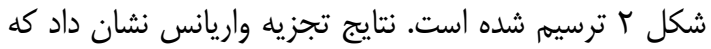

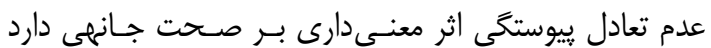

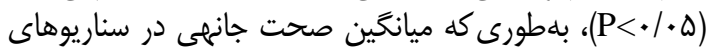

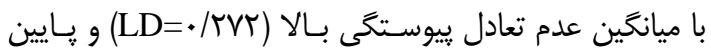

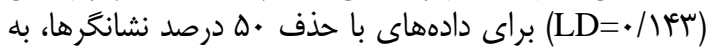

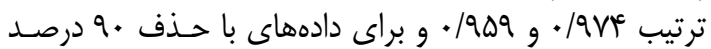

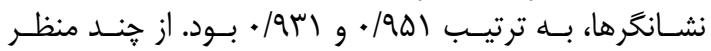

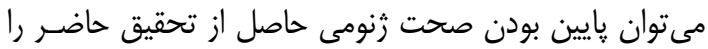

جدول ז- ميانگين و انحر اف استاندارد عدم تعادل بيوستخى و صحت جانهى بين زنوتيڤهاى اصلى و جانهى شده در سناريوهاى مختلف Table 2. Mean and standard deviation (in bracket) of linkage disequilibrium and imputation accuracy between imputed and original genotypes in different scenarios

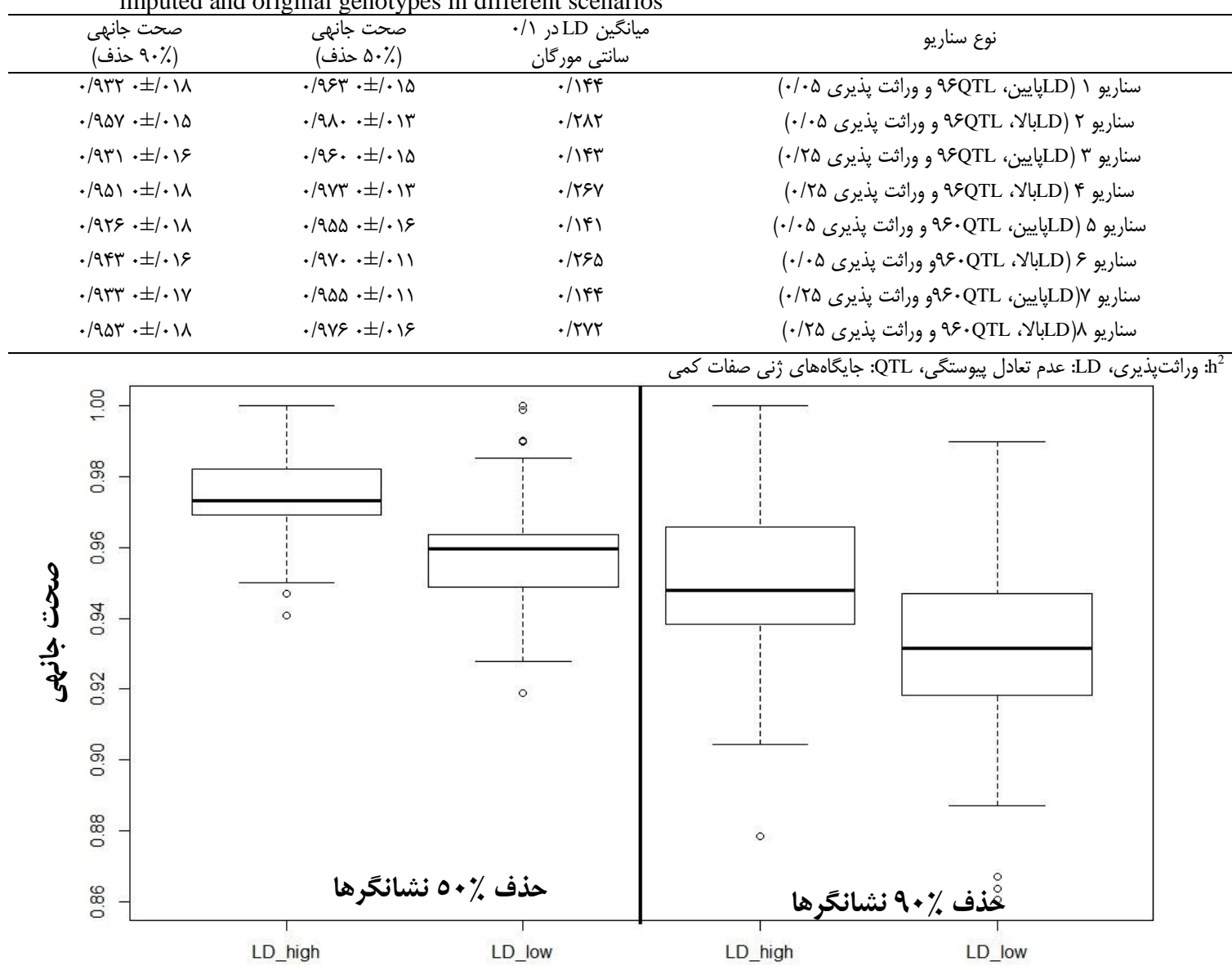

شكل r- نمودار جعبهاى، صحت جانمى براى سطوح مختلف عدم تعادل يبيوستخى

Figure 2. The box-plots of imputation accuracy for the different levels of linkage disequilibrium. 


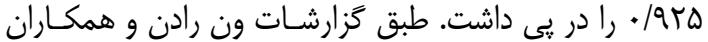

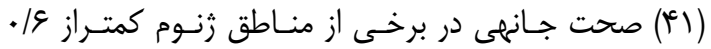

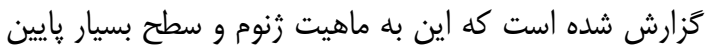

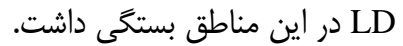

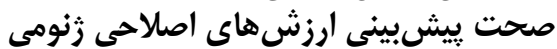

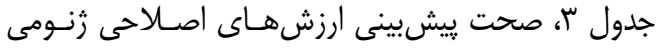

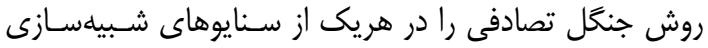
شده (اصلى و جانهى) براى صـفات كمى وآسـتانهاى نشـان مى دهد.

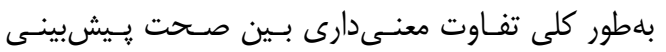

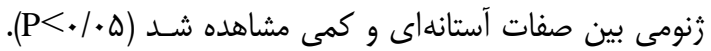

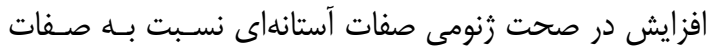

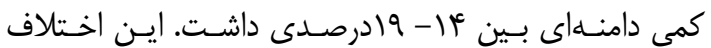

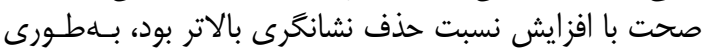

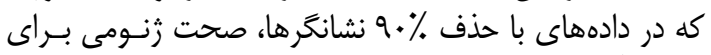

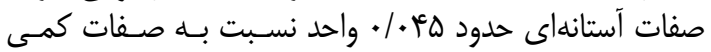

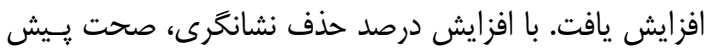

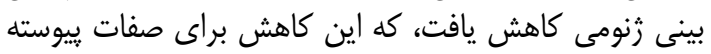

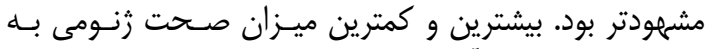

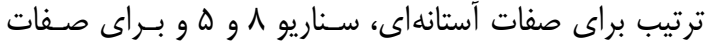

$$
\text { كمى، سناريو أ و ه بود. }
$$

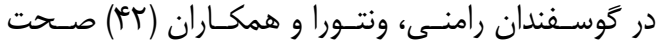

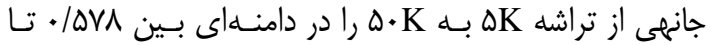

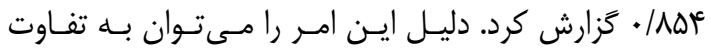

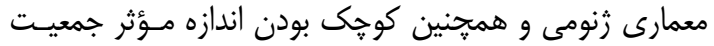

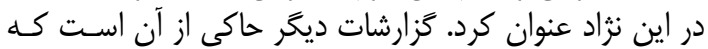

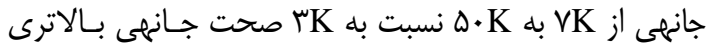

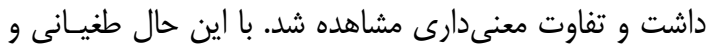

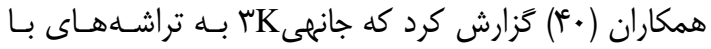

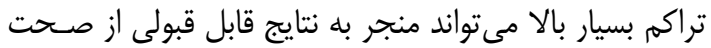

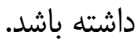

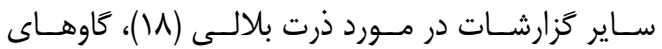

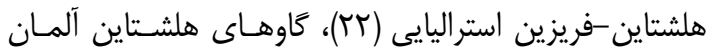

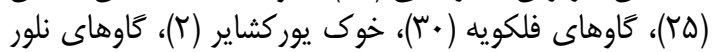

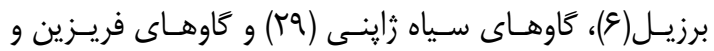

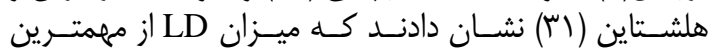

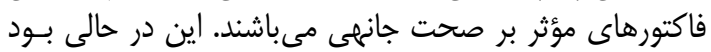

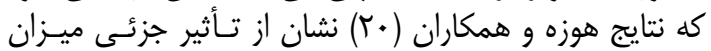

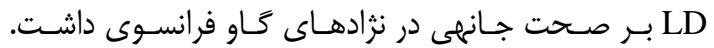

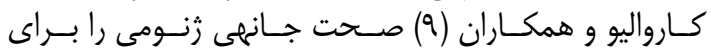

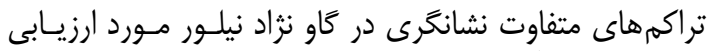

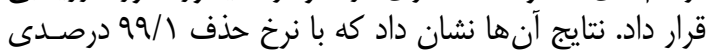

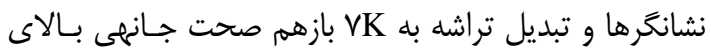

جدول ب- ميانگين و انحراف استاندارد صحت ييشبينى ارزشهاى اصلاحى زنومى در دادهاى اصلى و جانهى با استفاده از روش جنگل تصادفى

Table 3. Mean and standard deviation of GEBVs accuracies by random forest method in the original and imputed SNP genotypes

\begin{tabular}{|c|c|c|c|c|c|c|}
\hline \multicolumn{3}{|c|}{ صحت زنومى صفات كمى } & \multicolumn{3}{|c|}{ صحت زنومى صفات آستانهاى } & \multirow[b]{2}{*}{ شماره سناريو } \\
\hline حذف \% •9 نشانخرها & حذف \% ه نشانگرها & اصلى & حذف \%٪.9 نشانخرها & حذف \% ه نشانخرها & اصلى & \\
\hline$\cdot / 19 t \cdot \pm / \cdot r$ & $\cdot / 191 \cdot \pm / \cdot r$ & $\cdot / 4 \mid T_{f} \cdot \pm / \cdot 1$ & $\cdot / 190 \cdot \pm / \cdot r$ & $\cdot / r \mid \Lambda \cdot \pm / \cdot r$ & $\cdot /$ T\&E. $\pm / \cdot 1$ & 1 \\
\hline ./trt. $\pm / \cdot r$ & . & $\cdot / T \Delta V \cdot \pm / \cdot r$ & •/TND $\cdot \pm / \cdot r$ & $\cdot / r \cdot r \cdot \pm / \cdot r$ & $\cdot / \mu I f \cdot \pm / \cdot r$ & r \\
\hline س. & 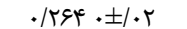 & $. / r q q \cdot \pm / \cdot r$ & ./rqץ $\cdot \pm / \cdot r$ & . & . & r \\
\hline 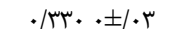 & $\cdot|r \Delta| \cdot 7 / \cdot r$ & ( & $\cdot|r| m|\cdot| \cdot r$ & $\cdot / r \Delta q \cdot \pm / \cdot r$ & ( & f \\
\hline$\cdot / \mathrm{TN} \cdot \pm / \cdot r$ & $\cdot / \backslash \Delta \varepsilon \cdot \pm / \cdot r$ & $\cdot / \backslash \wedge \Delta \cdot \pm / \cdot r$ & $\cdot / I V^{e} \cdot \pm / \cdot r$ & $\cdot / 191 \cdot \pm / \cdot r$ & $\cdot / r T \Delta \cdot \pm / \cdot r$ & $\Delta$ \\
\hline$\cdot / t \cdot r \cdot \pm / \cdot r$ & $\cdot|t 4| \cdot \pm 1 \cdot 1$ & (1) & ./rtg. $7 / \cdot r$ & $\cdot / T \Delta \cdot \cdot \pm / \cdot r$ & ./TER $\cdot \pm / \cdot r$ & 8 \\
\hline מ./ATt & $\cdot / t e d \cdot \pm / \cdot r$ & $\cdot / T V E \cdot \pm / \cdot r$ & •/TVD • & $\cdot|r q| \cdot 7 / \cdot r$ & (ז/ & $\checkmark$ \\
\hline$\cdot / r q q \cdot \pm / \cdot r$ & $\cdot / r \mid r \cdot \pm / \cdot r$ & 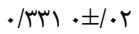 & & $\cdot / r+\cdots+ \pm / \cdot r$ & $\cdot|k t| \cdot \pm / \cdot 1$ & $\wedge$ \\
\hline
\end{tabular}

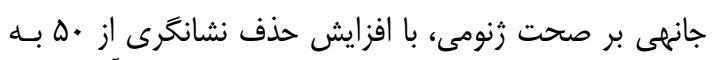

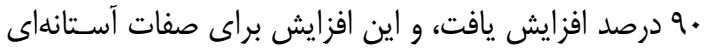

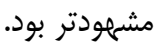

بررسى مـدل رگرسـيونى تحقيـق حاضـر نشـان داد كـهـ

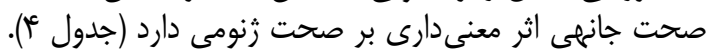

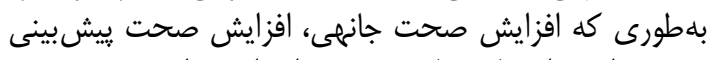

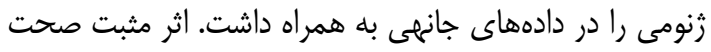

Table 4. Effect of imputation accuracy on accuracy of genomic prediction

جدول ع - اثر صحت جانهى بر صحت ييشبينى زنومى

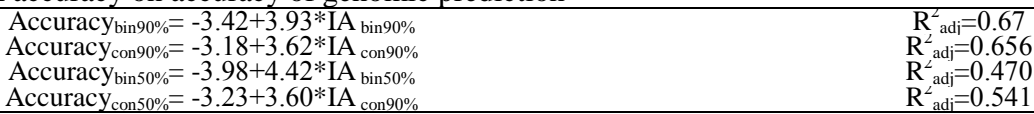

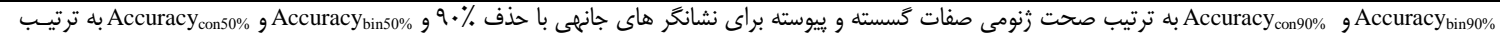

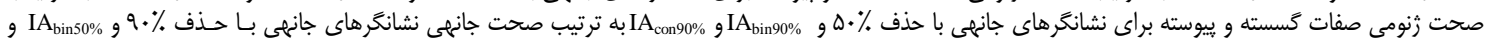
IA به ترتيب صحت جانهى 


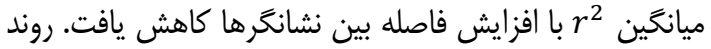

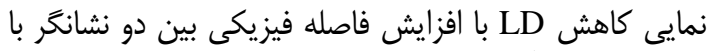

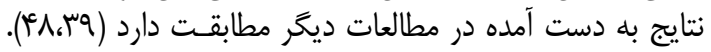

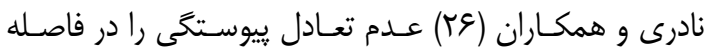

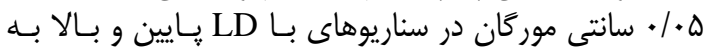

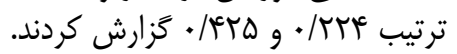

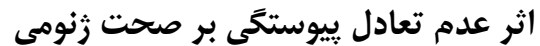

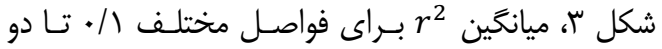

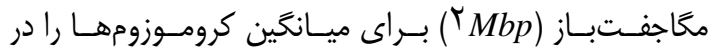

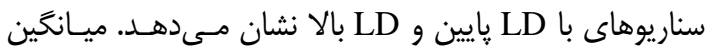

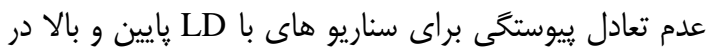

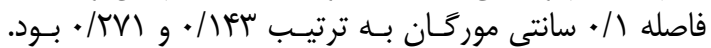

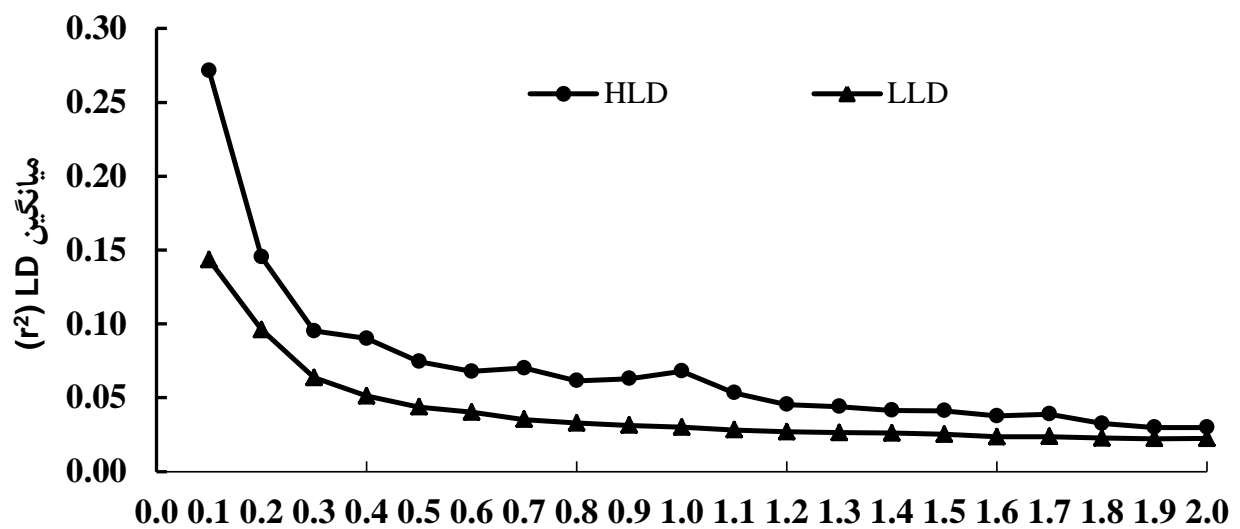

فاصله بين دو نشانخر (Mbp)

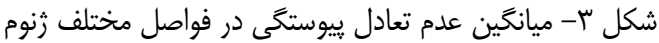

Figure 3. Mean of linkage disequilibrium in different intervals of the genome

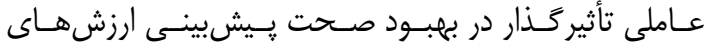
اصلاحى زنومى عنوان كرد.

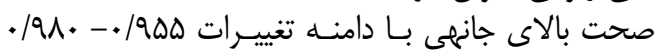

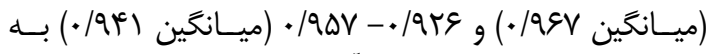

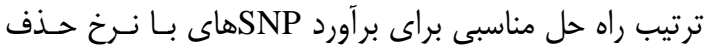

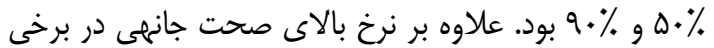

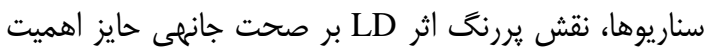

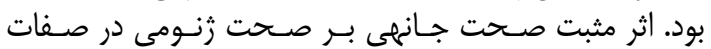

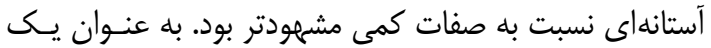

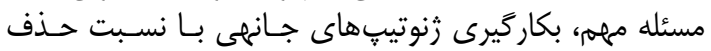

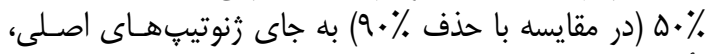

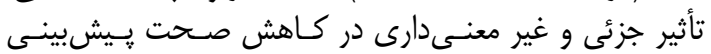

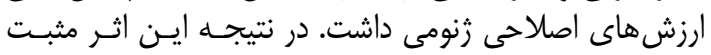

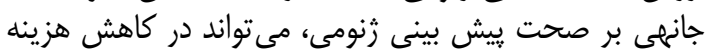
رنومى مؤثر باشد.

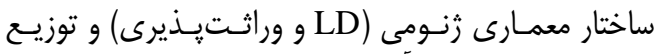

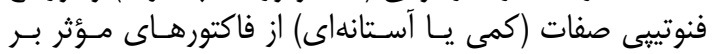

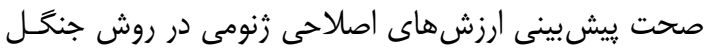

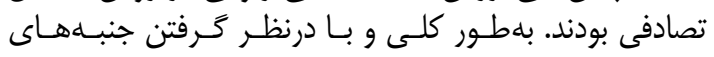

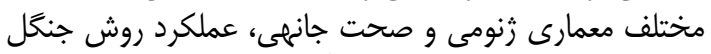

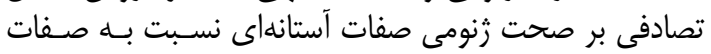
ييوسته برترى داشت.

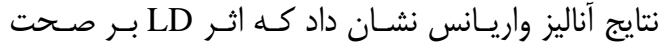

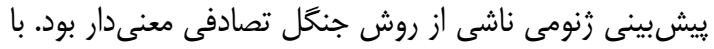

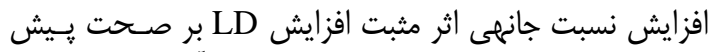

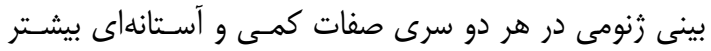

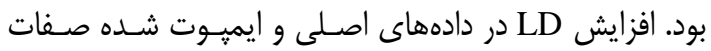

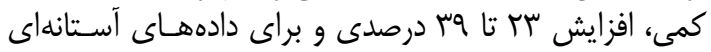

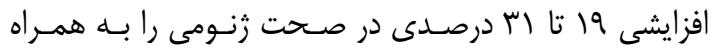

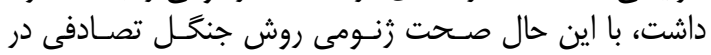

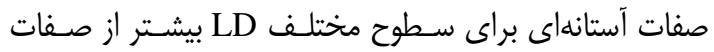

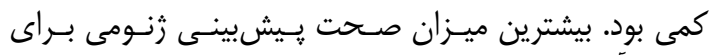

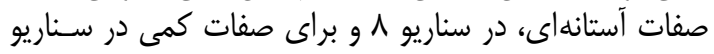

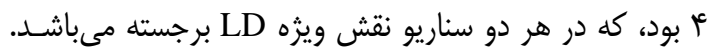

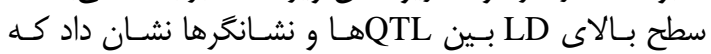

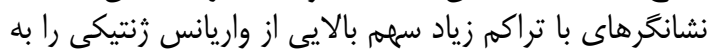

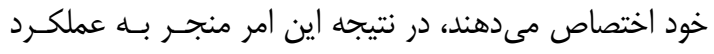

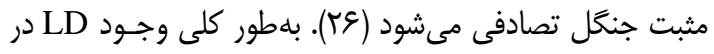

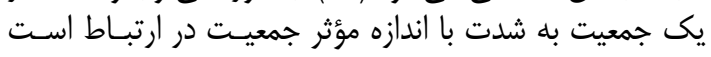

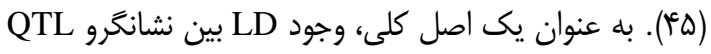

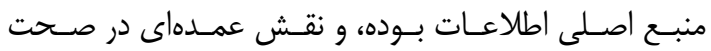

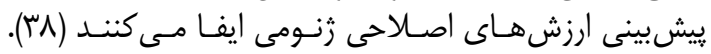

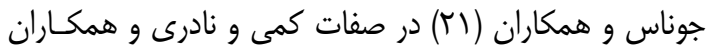

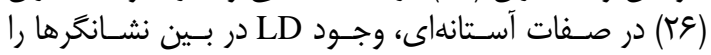


1. Atefi, A., A.A. Shadparvar, and N.G. Hossein-Zadeh. 2016. Comparison of whole genome prediction accuracy across generations using parametric and semi parametric methods. Acta Scientiarum. Animal Sciences, 38(4): 447-453.

2. Badke, Y.M., R.O. Bates, C.W. Ernst, J. Fix and J.P. Steibel. 2014. Accuracy of estimation of genomic breeding values in pigs using low-density genotypes and imputation. G3: Genes Genomes Genetics, 4(4): 623-631.

3. Baneh, H., A. Nejati-Javaremi, G. Rahimi-Mianji and M. Honarvar. 2017. Genomic evaluation of threshold traits with different genetic architecture using bayesian approaches. Research on Animal Production, 8: 149-154 (In Persian).

4. Beckmann, J. and M. Soller. 1983. Restriction fragment length polymorphismsin genetic improvement: methodologies, mapping and costs. Theoretical and Applied Genetics, 67(1): 35-43.

5. Bo, Z., J.J. Zhang, N. Hong, G. Long, G. Peng, L.Y. Xu, C. Yan, L.P. Zhang, H.J. Gao and G. Xue. 2017. Effects of marker density and minor allele frequency on genomic prediction for growth traits in Chinese Simmental beef cattle. Journal of Integrative Agriculture, 16(4): 911-920.

6. Boison, S., H.H. Neves, A.P.O. Brien, Y.T. Utsunomiya, R. Carvalheiro, M.da Silva, J. Solkner and J.F. Garcia. 2014. Imputation of non-genotyped individuals using genotyped progeny in Nellore, a Bos indicus cattle breed. Livestock Science, 166:176-189.

7. Breiman, L. 2001. Random forests. Machine learning, 45(1): 5-32.

8. Browning, S.R. 2008. Missing data imputation and haplotype phase inference for genome-wide association studies. Human genetics, 124(5): 439-450.

9. Carvalheiro, R., S.A. Boison, H.H. Neves, M. Sargolzaei, F.S. Schenkel, Y.T. Utsunomiya, A.M.P. Obrien, J. Solkner, J.C. McEwan, and C.P. Van Tassell. 2014. Accuracy of genotype imputation in Nelore cattle. Genetics Selection Evolution, 46(1): 69.

10. Chen, L., C. Li, M. Sargolzaei and F. Schenkel. 2014. Impact of genotype imputation on the performance of GBLUP and Bayesian methods for genomic prediction. PLoS One, 9(7): e101544.

11. Clark, S.A., J.M. Hickey and J.H. Van der Werf. 2011. Different models of genetic variation and their effect on genomic evaluation. Genetics Selection Evolution, 43(1): 18.

12. Ghafouri-Kesbi, F., G. Rahimi-Mianji, M. Honarvar and A. Nejati-Javaremi. 2017. Predictive ability of random forests, boosting, support vector machines and genomic best linear unbiased prediction in different scenarios of genomic evaluation. Animal Production Science, 57(2): 229-236.

13. Ghafouri-Kesbi, F., G. Rahimi-Mianji, M. Honarvar, and A. Nejati-Javaremi. 2016. Tuning and application of random forest algorithm in genomic evaluation. Research on Animal Production, 7 (13): 178-185 (In Persian).

14. Goddard, M. and B. Hayes. 2007. Genomic selection. Journal of Animal breeding and Genetics, 124(6): 323-330.

15. Goddard, M.E. and B.J. Hayes. 2009. Mapping genes for complex traits in domestic animals and their use in breeding programmes. Nature Reviews Genetics, 10(6): 381-391.

16. Gonzalez-Recio, O. and S. Forni. 2011. Genome-wide prediction of discrete traits using Bayesian regressions and machine learning. Genetics Selection Evolution, 43(1): 7.

17. Hayes, B. and M.E. Goddard. 2001. The distribution of the effects of genes affecting quantitative traits in livestock. Genetics Selection Evolution, 33: 209.

18. Hickey, J.M., J. Crossa, R. Babu and G. de los Campos. 2012. Factors affecting the accuracy of genotypeimputation in populations from several maize breeding programs. Crop Science, 52(2): 654663.

19. Hill, W. and A. Robertson. 1968. Linkage disequilibrium in finite populations. TAG Theoretical and Applied Genetics, 38(6): 226-231.

20. Hoze, C., M.N. Fouilloux, E .Venot, F. Guillaume, R. Dassonneville, S. Fritz, V. Ducrocq, F. Phocas, D. Boichard and P. Croiseau. 2013. High-density marker imputation accuracy in sixteen French cattle breeds. Genetics Selection Evolution, 45(1): 33.

21. Jonas, D., V. Ducrocq and P. Croiseau. 2017. The combined use of linkage disequilibrium-based haploblocks and allele frequency-based haplotype selection methods enhances genomic evaluation accuracy in dairy cattle. Journal of Dairy Science, 100(4): 2905-2908.

22. Khatkar, M.S., G. Moser, B.J. Hayes and H.W. Raadsma. 2012. Strategies and utility of imputed SNP genotypes for genomic analysis in dairy cattle. BMC genomics, 13(1): 538 .

23. Li, Y., J. Kijas, J. Henshall, S. Lehnert, R. McCulloch, and A. Reverter. 2014. Using random forests (RF) to prescreen candidate genes: A new prospective for GWAS. in Proc. Proc. of $10^{\text {th }}$ World Congress for Genetics Applied to Livestock Production, British Columbia, Vancouver.

24. Meuwissen, T., B. Hayes and M. Goddard. 2001. Prediction of total genetic value using genome-wide dense marker maps. Genetics, 157(4): 1819-1829.

25. Mulder, H., M. Calus, T. Druet and C. Schrooten. 2012. Imputation of genotypes with low-density chips and its effect on reliability of direct genomic values in Dutch Holstein cattle. Journal of dairy science, 95(2): 876-889.

26. Naderi, S., T. Yin and S. Konig. 2016. Random forest estimation of genomic breeding values for disease susceptibility over different disease incidences and genomic architectures in simulated cow calibration groups. Journal of Dairy Science, 99(9): 7261-7273.

27. Neves, H.H., R. Carvalheiro and S.A. Queiroz. 2012. A comparison of statistical methods for genomic selection in a mice population. BMC genetics, 13(1): 100.

28. Nguyen, T.T., J.Z. Huang, Q. Wu, T.T. Nguyen and M.J. Li. 2015 .Genome-wide association data classification and SNPs selection using two-stage quality-based Random Forests. Page S5 in Proc. BMC genomics. BioMed Central Ltd. 
IIV

29. Ogawa, S., H. Matsuda, Y. Taniguchi, T. Watanabe, A. Takasuga, Y. Sugimoto and H. Iwaisaki. 2016. Accuracy of imputation of single nucleotide polymorphism marker genotypes from low-density panels in Japanese Black cattle. Animal Science Journal, 87(1): 3-12.

30. Pausch, H., B. Aigner, R. Emmerling, C. Edel, K.U. Götz and R. Fries. 2013. Imputation ofhighdensity genotypes in the Fleckvieh cattle population. Genetics Selection Evolution, 45(1): 3.

31. Pausch, H., I.M. MacLeod, R. Fries, R. Emmerling, P.J. Bowman, H.D. Daetwyler and M.E. Goddard. 2017. Evaluation of the accuracy of imputed sequence variant genotypes and their utility for causal variant detection in cattle. Genetics Selection Evolution, 49(1): 24.

32. Pimentel, E., C. Edel, R. Emmerling and K.U. Gotz. 2015. How imputation errors bias genomic predictions. Journal of dairy science, 98(6): 4131-4138.

33. Purcell, S., B. Neale, K. Todd-Brown, L. Thomas, M.A. Ferreira, D. Bender, J. Maller, P. Sklar, P.I. De Bakker and M.J. Daly. 2007. PLINK: a tool set for whole-genome association and populationbased linkage analyses. The American Journal of Human Genetics, 81(3): 559-575.

34. Saatchi, M., J. Ward and D. Garrick. 2013. Accuracies of direct genomic breeding values in Hereford beef cattle using national or international training populations. Journal of animal science, 91(4): 15381551.

35. Sargolzaei, M., J. Chesnais and F. Schenkel. 2011. FImpute-An efficient imputation algorithm for dairy cattle populations. Journal of Dairy Science, 94(1): 421-422.

36. Sargolzaei, M. and F.S. Schenkel. 2009. QMSim: a large-scale genome simulator for livestock. Bioinformatics, 25(5): 680-681.

37. Solberg, T., A. Sonesson and J. Woolliams. 2008. Genomic selection using different marker types and densities. Journal of animal science, 86(10): 2447-2454.

38. Sun, X., R. Fernando and J. Dekkers. 2016. Contributions of linkage disequilibrium and cosegregation information to the accuracy of genomic prediction. Genetics Selection Evolution, 48(1): 77.

39. Thomasen, J.R. 2013. Genomic selection in small dairy cattle populations. Aarhus UniversitetAarhus University, Science and TechnologyScience and Technology, Institut for Molekylærbiologi og GenetikDepartment of Molecular Biology and Genetics, Institut for Molekylærbiologi og GenetikCenter for Kvantitativ Genetik og Genomforskning Department of Molecular Biology and GeneticsCenter for Quantitative Genetics and Genomics.

40. Toghiani, S., S. Aggrey and R. Rekaya. 2016. Multi-generational imputation of single nucleotide polymorphism marker genotypes and accuracy of genomic selection. animal, 10(7): 1077-1085.

41. VanRaden, P., D. Null, M. Sargolzaei, G. Wiggans, M. Tooker, J. Cole, T. Sonstegard, E. Connor, M. Winters, and J. van Kaam. 2013. Genomic imputation and evaluation using high-density Holstein genotypes. Journal of dairy science, 96(1): 668-678.

42. Ventura, R.V., S.P. Miller, K.G. Dodds, B. Auvray, M. Lee, M. Bixley, S.M. Clarke and J.C. McEwan. 2016. Assessing accuracy of imputation using different SNP panel densities in a multi-breed sheep population. GeneticsSelection Evolution, 48(1): 71.

43. Villumsen, T., L. Janss and M. Lund. 2009. The importance of haplotype length and heritability using genomic selection in dairy cattle. Journal of Animal Breeding and Genetics, 126(1): 3-13.

44. Visscher, P. and C. Haley. 1998. Strategies for marker-assisted selection in pig breeding programmes. Stočarstvo, 52(6): 425-434.

45. Wang, Q., Y. Yu, J. Yuan, X. Zhang, H. Huang, F. Li and J. Xiang. 2017. Effects of marker density and population structure on the genomic prediction accuracy for growth trait in Pacific white shrimp Litopenaeus vannamei. BMC genetics, 18(1): 45.

46. Wang, Y., G. Lin, C. Li and P. Stothard. 2016. Genotype Imputation Methods and Their Effects on Genomic Predictions in Cattle. Springer Science Reviews, 4(2): 79-98.

47. Wientjes, Y.C., R.F. Veerkamp, P. Bijma, H. Bovenhuis, C. Schrooten and M.P. Calus. 2015. Empirical and deterministic accuracies of across-population genomic prediction. Genetics Selection Evolution, 47(1): 5 .

48. Yin, T., E. Pimentel, U.K.V. Borstel and S. Konig. 2014. Strategy for the simulation and analysis of longitudinal phenotypic and genomic data in the context of a temperaturex humidity-dependent covariate. Journal of dairy science, 97(4): 2444-2454. 


\title{
Evaluation of Genomic Prediction Accuracy in Different Genomic Architectures of Quantitative and Threshold Traits with the Imputation of Simulated Genomic Data using Random Forest Method
}

\author{
Yousef Naderi
}

Assistant Professor, Department of Animal Science, Astara Branch, Islamic Azad University, Astara, Iran (Corresponding author: y.naderi@iau-astara.ac.ir) Received: January 15, 2018

Accepted: June 11, 2018

\begin{abstract}
Genomic selection is a promising challenge for discovering genetic variants influencing quantitative and threshold traits for improving the genetic gain and accuracy of genomic prediction in animal breeding. Since a proportion of genotypes are generally uncalled, therefore, prediction of genomic accuracy requires imputation of missing genotypes. The objectives of this study were (1) to quantify imputation accuracy and to assess the factors affecting it; and (2) to evaluate the genomic accuracy of random forest (RF) algorithm to analyze binary threshold and quantitative traits. In the first phase, genomic data were simulated by QMSim software to reflect variations in heritability $\left(\mathrm{h}^{2}=0.05\right.$ and 0.25$)$, number of QTL (QTL=96 and 960) and linkage disequilibrium ( $\mathrm{LD}=$ low and high) for 48 chromosomes. In the second phase, for real condition simulating, we randomly masked markers with $50 \%$ and $90 \%$ missing rate for each scenario; afterwards, hidden markers were imputed using FImpute software, and estimated imputation accuracy. In the third phase, to estimate genomic breeding values, we applied Random forest algorithm for original (before masking a proportion of SNPs) and imputed genotypes with quantitative and quality phenotypes. The accuracy of imputation was improved with increasing level of LD. With increase a major proportion of masked markers $(90 \%)$, results of current study shed light on the effects of imputation accuracy on accuracy of genomic prediction. In the scenario combining the highest heritability, LD and QTL for threshold traits and in the scenario combining the highest heritability and LD and the least QTL for quantitative traits, random forest method had the best performance of genomic accuracy. Generally, accuracy of genomic prediction for threshold traits had more precise than quantitative trait when using the random forest method.
\end{abstract}

Keywords: Linkage disequilibrium, Discrete traits, Machine learning, Imputation, Genomic architecture 